\title{
Distal pancreatectomy with celiac artery resection (DP-CAR) \& portal vein resection as \\ a conversion surgery after neoadjuvant chemotherapy for pancreatic adenocarcinoma
}

\author{
Yang Won $\mathrm{NAH}^{* 1}$, Yun Beom RYU' ${ }^{2}$, Eun Ji LEE', Sang Min KONG', Jaekyung CHEON ${ }^{3}$ \\ 'Department of Surgery, Ulsan University Hospital, Ulsan, Korea \\ ${ }^{2}$ Department of Surgery, Asan Medical Center, Seoul, Korea \\ ${ }^{3}$ Department of Hemato-Oncology, Ulsan University Hospital, Ulsan, Korea
}

Introduction: The authors performed distal pancreatectomy with celiac artery resection (DP-CAR) \& portal vein (PV) resection and reconstruction with allogeneic iliac vein as a conversion surgery after neoadjuvant chemotherapy for locally advanced pancreas cancer (LAPC) and report here with video.

Methods: A 58-year-old man presented with LAPC. There was a $4 \mathrm{~cm}$ sized tumor at pancreas body abutting celiac axis, superior mesenteric artery, common hepatic artery, splenic artery and portosplenic confluence. There was no evidence of distant metastasis. Modified FOLFIRINOX was given for 17 cycles per 8 months. The tumor shrunk to $3.2 \mathrm{~cm}$ with markedly decreased area of abutment to major vasculatures.

Results: The DP-CAR procedures were proceeded as planned except portosplenic confluence management where resection and reconstruction with an allogeneic iliac vein graft of $4 \mathrm{~cm}$ length was performed. The operation took 355 minutes. Intraoperatively blood loss amounted $900 \mathrm{~g}$ with no transfusion. R0 resection was achieved pathologically. There was one lymph node metastasis. Postoperatively infected fluid collection due to pancreas leakage was identified and percutaneous drainage was inserted 3 weeks after the operation. PV graft was stenotic due to the fluid collection. PV stent was inserted. PCD tube was removed 6 weeks after its insertion. He is well except diarrhea 3 times a day 3 months after the operation.

Conclusions: DP-CAR with PV resection and reconstruction is a demanding operation. PV reconstruction should be anticipated and prepared before surgery when pancreas body cancer abuts portosplenic confluence. Interposition graft rather than primary anastomosis should be considered when PV resection is needed during DP-CAR. 